\title{
8 Metal Gear Solid and its comics adaptations
}

\author{
Claudius Stemmler
}

Both popular discourse and academic scholarship have a tendency to consider the relationship between comics and videogames primarily from the angle of comics as the source for various elements in videogames. This is not surprising because, as a multimodal medium (Ryan and Thon 2014, 11), videogames are well suited to encompass elements from other media formats. Moving into the opposite direction, comics adaptations have to transpose complex multimodal systems into a combination of images and words. Perhaps this complexity has lessened their appeal since comics adaptations have, similar to film novelizations, rarely received scholarly attention and appear to be mostly treated as a mere side effect of today's integrated media industries. This, however, ignores the fact that such adaptations, independent of their artistic value, possess great potential to explore the relationship between comics and videogames as well as each medium's individual properties.

In 1998, the Japanese company Konami released Metal Gear Solid for the Sony PlayStation "to critical acclaim and commercial success" (Parkin 2012, n.p.). The game represented not only "an enormous leap forward in terms of what a story-driven action game could be" (Parish 2018, n.p.), but it also "formalize[d] and popularize[d] 'Stealth' as a kind of genre" (Parkin 2017, 147). Three years later, a sequel named Metal Gear Solid 2: The Sons of Liberty (2001) was released for the Sony PlayStation 2. Despite the fact that the game's narrative lead to a backlash (Holmes 2012, 135) and its "divisive nature clearly drove away a portion of the audience" (Holmes 2012, 144), it was a commercial success, with its ludic elements very much fulfilling expectations toward an improved sequel (Holmes 2012, 134). In 2004, the US-based company IDW Publishing began releasing a Metal Gear Solid comics series. Running for 24 issues, the series adapted both Metal Gear Solid (Oprisko and Wood 2014) and its sequel (Fraction and Wood 2014; Garner and Wood 2014). In a highly unusual move, Konami itself then began releasing further adaptations of these comics. Calling them digital graphic novels ${ }^{1}$ and releasing them for the Sony PlayStation Portable (in 2006) and as feature films on DVD (in 2008), these adaptations reproduced the comics' narrative while taking advantage of their digital platforms' 
audiovisual capabilities. Taken together, this group of releases constitutes a unique object to study the relationship between videogames and comics.

In this chapter, I will analyze Metal Gear Solid, its sequel, and the aforementioned adaptations. These adaptations into linear narrative media are particularly intriguing because the reception of both games highlighted their ludic as well as their narrative elements. Alongside the question of how their multimodal aesthetics have been adapted, this invites asking if and how their distinctive ludic nature has been transposed. The first section of this chapter therefore analyzes the narration of these various incarnations while the second section examines their presentation. In both cases, this will reveal how their respective media format's properties shaped these elements. The conclusion presents an outlook on noticeable developments following this particular sequence of adaptations.

\section{Narration and narrative}

A general trait of adaptations is that they have "an overt and defining relationship to [a] prior tex[t]" (Hutcheon 2006, 3-4). At the same time, the broad range of existing adaptations makes it difficult to present a more specific definition of this relationship without losing its general applicability. Typically, theories of adaptation have assumed this relationship to center around the shared core of a work's narrative (Hutcheon 2006, 10). This assumption might, however, be more indebted to the fact such adaptations are, perhaps inadvertently, prioritized by scholars because they fit more easily into existing humanities frameworks. It is at this point that videogames as a source for adaptations are intriguing thanks to their contested narrativity. While the so-called ludology-narratology debate ended with blanket statements regarding videogames' narrativity being replaced by more moderate approaches acknowledging their potential narrativity (Mukherjee $2015,7)$, its existence also highlighted the complexity of the issue. Analyzing adaptations of videogames into linear narrative media therefore begs the question of how their elements outside of narrative frameworks have been transposed. Does the process of adaptation resemble the extraction of a linear narrative from preexisting elements, or is it rather an addition of elements in order to create a coherent narration?

\section{The games}

In his essay "In Defense of Cutscenes," Rune Klevjer uses Metal Gear Solid as an example of a game that radical ludologists would consider "an artistic failure" $(2002,194)$. This is because a distinctive trait shared by the game and its sequel is the strong presence of embedded ${ }^{2}$ narrative content. In both games, the process of playing is shaped by a structural interplay between three modes, gameplay, cutscenes, and codec communication (Holmes 2012, $86),{ }^{3}$ of which only one allows players to engage with the game's rule-based 
systems. The other two solely consist of embedded narrative elements whose presentation resembles earlier media formats. ${ }^{4}$ Cutscenes are modeled in the audiovisual language of film, while codec communication with its sparse visual presentation accompanying voiced dialogue resembles radio drama (Holmes 2012, 86). Typically, the game switches between gameplay and these modes at predetermined points. Additionally, the player can actively switch from gameplay to codec communication to initiate a conversation with selected characters. While the avatar's specific situation influences which conversation occurs, the conversations themselves are linear. In this way, the player is granted access to a large number of optional embedded narrative segments. In the same vein, the game also features a small number of optional cutscenes triggered by circumstances not necessarily fulfilled during a playthrough. However, aside from these instances, both modes present a fixed sequence of diegetic events in which the player's position as recipient is akin to linear narratives.

In contrast, during gameplay the player engages with the games' rulebased systems through the avatar. The game's core mechanics (Salen and Zimmerman 2008, 317) constitute the avatar's abilities; they are applied to avoid detection while traversing the diegetic world. Furthermore, when detected and at predetermined points, the avatar has to fight his enemies. Besides these recurring elements, gameplay also encompasses singular events such as the avatar in Metal Gear Solid being subjected to torture. Typically, gameplay situations are emergent (Juul 2005, 73) and can be completed in various ways. However, aside from the torture sequence, the specific solution does not influence later embedded diegetic events. Together with the limited diversity of the emergent narrative events presented through gameplay, this might incline us to attribute to this mode a superficial role in terms of narration. This inclination, however, would ignore the narrative elements present in gameplay, which, similar to a film's mise-en-scène, often fail to be encompassed by models of linear narration. First, gameplay turns the diegetic world in a spatially realized fictional space full of narrative descriptors (Salen and Zimmerman 2008, 404) the player can explore through the avatar. Second, the game's rule-based systems constitute a procedural rhetoric (Bogost 2007, 3) in support of their thematic treatments. Gameplay also helps create an oscillating relationship between the avatar and the players as they switch between watching and performing as their digital embodiment (Backe 2008, 344). In both games, this relationship is effectively employed as part of the narration. Together, the three modes constitute a cycle in which embedded narrative events presented through cutscenes and codec communication introduce diegetic goals that the player's avatar then pursues in gameplay.

For the most part, the games' sequences of embedded narrative events are fixed and remain identical between playthroughs. There are, however, three noticeable exceptions. First, the aforementioned torture sequence in Metal Gear Solid, where the outcome decides which ally will later accompany 
the avatar. Not merely changing their sequential presentation, players here directly influence which of two embedded narrative endings will take place. Another exception is the optional briefing section in Metal Gear Solid. Here, the player can navigate a branching structure where some segments present the avatar being briefed through voiced dialogue and sparse animation while others consist solely of written text. While the player has control over the flow of narrative information, this does not influence the diegetic world's sequence of events. The same is true for the division of Metal Gear Solid 2: Sons of Liberty into two separately selectable chapters. In contrast to the optional briefing section, this presents a highly distinctive feature of modifying the diegetic events' sequential presentation. This is particularly noticeable in the game's original release, where only players acknowledging to be familiar with Metal Gear Solid played both chapters. What is remarkable about all these exceptions is that they strongly modify the player's reception through technologically simple means.

In summary, due to the strong presence of fixed sequences of embedded narration, both games generally fit well into classic models of narration. At the same time, they also feature various elements through which their narrative presentation exceeds the potential of established linear narrative media. An apt description of the process of playing would include the oscillation between classical textual and distinctively ludic elements, which is a structure typical of narrative videogames (Backe 2008, 109).

\section{The comics}

The specific choice of adapting Metal Gear Solid and Metal Gear Solid 2: Sons of Liberty into comics appears distinctly twofold. On the one hand, they appear as intuitive choices thanks to their large amount of embedded narrative elements set in a fixed sequence. On the other hand, both games feature several distinctive elements that cannot be replicated within a linear narrative presentation. Because of the relation of this dichotomy to the games' structured interplay, it is perhaps not surprising that the comics, despite not replicating this structured interplay, still appear distinctively shaped by it.

This is particularly evident in the comics version of Metal Gear Solid, where most of the panels are encapsulated visuals retraced from cutscenes. However, the source of these visuals is not Metal Gear Solid but the game's remake Metal Gear Solid: The Twin Snakes (2004). ${ }^{5}$ This remake featured revised cutscenes that depict more flamboyant physical action, thereby moving the comic's presentation closer to the superhero genre. Furthermore, only in-game engine cutscenes were adapted while those employing film footage have been omitted. Through their dialogue and by presenting locations outside the closer diegetic setting, these cutscenes supported framing the game's fictional events within real-world circumstances. Their omission now makes the narrative of the comic appear not only more 
geographically enclosed but also more escapist than that of its source material. In comparison to cutscenes, only a small number of panels have been adapted from gameplay. Here, rather than concentrating on the game's core mechanic of sneaking, the focus lies on the protagonist's battles with various antagonists. The game's last mode, codec communication, has not been adapted as designated panels but rather as a distinctive set of speech balloons. Frequently, these have been added to panels adapted from gameplay where the game's presentation did not feature dialogue. Nonetheless, large parts of the game's dialogue presented through codec communication were dropped. This pattern of adaptation was generally retained for the comics version of Metal Gear Solid 2: Sons of Liberty. A major difference is, however, that more panels appear not directly retraced from the game's cutscenes. This goes hand in hand with the adaptation modifying the game's diegetic events to a larger degree, leading many panels to have no direct equivalent in the game. In general, the comics' pattern of adaptation can be described as extracting a narrative by concentrating on the games' elements suitable for linear narrative media. In its omission of large amounts of dialogue, this pattern highlights the visual primacy of comics. Furthermore, it also reveals the games' partial detachment between their narrative and their ludic core mechanic. Particularly the first games' adaptation shows that, even while only sparsely representing the sneaking mechanic, the narration remains coherent without adding new elements in its place.

As taken from their sources, the narratives of both comics adaptations could be described as twice restructured. First, a linear narrative was extracted from the games' potential variations. Then, a new layer of narrative structure was added through their episodic release. In Metal Gear Solid, the game's briefing section is partially adapted as the beginning of the narrative, and a combination of the game's two endings serves as the narrative's end. For Metal Gear Solid: Sons of Liberty, part of the game's first chapter follows a new prologue while more of its diegetic events are later presented through an intradiegetic retelling. Taking aside those changes necessarily facilitated by the transposition into a linear narrative, these decisions appear to lessen the risk of audiences being confused by the narration. Different from the games, where audiences might be expected to continue engaging with the work's ludic elements even when briefly losing interest in the narration, the comics lack such a "safety net." This lack can even present an economic risk because of the comics' episodic publishing, something that appears reflected in the work as with one exception each issue constitutes a single chapter ending on a cliffhanger-like situation. Typically, the protagonist has either received new information that will change the reader's understanding of previous events, or he is standing on the brink of a confrontation. Aside from the economic reality of publishing, this structure also reveals how the narratives of the games, even without explicit markers, have a typical videogame structure. The protagonists move from one short-term goal to the next (Salen and Zimmerman 2008, 343), with each goal supplying 
rewards such as information or equipment. The adaptations make this structure more clearly recognizable as many of the games' elements that have disguised it have been omitted.

The adaptations also present different relationships among the reader, the protagonist, and the text itself. By necessity refraining from replicating the games' oscillating player-avatar relationship, the comics also create greater distance between the recipient's and the protagonist's range of narrative knowledge. The games generally tie the recipient's knowledge to the protagonist, with only cutscenes sporadically featuring a more omniscient range. Compared to this, the comics regularly present diegetic events outside of the protagonist's presence. This greater distance becomes particular noticeable in Metal Gear Solid: Sons of Liberty, where the adaptation's restructuring of the narration leads to two characters functioning as protagonists. While many diegetic events have been remodeled to feature greater participation by returning protagonist Solid Snake, other events still necessarily center on newcomer Raiden. This change is distinctive because the game's narration did heavily build on the identification between the player and rookie operative Raiden. In the same mold, the adaptations also change the relationship between the text and the recipient by removing the games' various postmodern elements, such as direct references to their own materiality. Not only heightening their appeal as escapist entertainment, this also leads to one major theme of Metal Gear Solid 2: Sons of Liberty being diminished in its adaptation.

In summary, the changes in the adaptations lead to a more escapist narrative with a heavier emphasis on physical action. In doing this, the comics retain much of the narrative core of the games and remain recognizable as adaptations. However, many of the omitted elements were important in creating the games' unique identity and in moving their narration beyond generic predecessors in various media. Their omission makes the comics' narratives appear more generic in comparison.

\section{The digital graphic novels}

As adaptations, a remarkable element of the Metal Gear Solid digital graphic novels is that they completely reproduce the comics' visual images and texts without any additions, omissions, or changes in their sequence. They differ, however, in their presentation of these elements. The digital graphic novels present each panel by itself before being replaced by the next one, which creates a stricter sequential order of reception. While the first release for the Sony PlayStation Portable allowed readers to select between manually switching panels or automated playback, the second option became the standard for all later releases, leading to an approximately film-like presentation. In both cases, the adaptations feature a more restrictive reception and thereby reveal the freedom granted to the reader in the comics' reception. Aside from the complete reproduction of the previously published comic's 
visual images and text, there is one additional narrative element in the Sony PlayStation Portable version of the digital graphic novel. Here, the recipient can not only switch between playback modes but can also pause playback to "scan" panels. By locating the right visual cues, this then expands the material accessible inside a database full of narrative background information. As a structure of supplying optional narrative information running alongside a linear narration, this feature recalls the games' player-initiated codec communication.

Despite including complete textual reproductions of their source material, the digital graphic novels present a different narrative experience. This makes them an ideal example of how the adaptation into another format necessarily facilitates changes even when its creators appeared to have tried to abstain from modifying the content.

\section{Presentation}

In terms of their presentation, a major difference distinguishing videogames from comics is that they are "an inherently multimodal medium" that frequently includes "haptics, visuals, music, spoken language, and written text" (Ryan and Thon 2014, 11). In this particular sequence of adaptations, the comics adaptations first had to transpose such a multimodal experience into a combination of images and words before the digital graphic novels restored many of the semiotic systems available to videogames, while merging them onto the comics' preexisting elements. In all of this, it is important to note that any kind of audiovisual presentation has to balance aesthetic and functional needs. In videogames, players have to make decisions based on their "reading" of the situation, and wrong decisions can stop narrative progress. In contrast, while the aesthetic functions of linear narrative media might frustrate the recipient's comprehension, they do not stop the narrative progress. Therefore, the relevance of such a balance is especially important when creating the audiovisual presentation of videogames.

\section{The games}

Metal Gear Solid was released at a time when technological developments were leading to frequent changes in the presentation of videogames. A noticeable trend, particular on console systems, was the combination of different styles of presentation. This was mainly facilitated by the introduction of the CD-ROM, which allowed the inclusion and playback of audiovisual elements in a quality the same hardware could not process in real-time. Because these elements were stored in a fixed state, their application lacked flexibility. Many games therefore mixed elements in various styles and qualities to optimize their balance between aesthetic appeal and ludic functionality.

This is also the case in Metal Gear Solid and Metal Gear Solid 2: Sons of Liberty, where the audiovisual presentation changes between their different 
modes. The games' most common form of presentation consists of polygonbased graphics, which are not only used for all of gameplay but also for all cutscenes depicting events inside the diegetic setting. ${ }^{6}$ Additionally, Metal Gear Solid 2: Sons of Liberty employs them for the character portraits in codec communication. During gameplay, the game's virtual camera typically presents a top-down viewpoint, with movements and additional angles dependent on context. While gameplay appears to prioritize the player's ability to oversee the avatar's situation, even here the games' approximation of cinematic aesthetics is recognizable in elements such as camera movements. The approximation of cinematic aesthetics is pushed further in cutscenes, where the games employ the audiovisual language of film while simultaneously removing visual markers of the games' rule-based systems. Throughout all of this, the visual assets are either identical or resemble those used in gameplay to a large degree. Aside from this, both games also feature stylistically different elements, such as animated and actual film footage in cutscenes and drawn character portraits during codec communication in Metal Gear Solid. Despite these exceptions, the general visual presentation appears in support of creating aesthetic coherence between the different modes. This is because, aside from the drawn character portraits, the use of film footage never presents the same characters and locations as the polygon-based graphics. Instead, these sequences generally connect the game's thematic treatments to real-world circumstances. Coupled with the games' general willingness to be self-referential, the stylistic difference appears appropriate, perhaps even supportive.

Another element enhancing the aesthetic coherence of the games is their aural presentation. The fact that all of the dialogue is performed by voice actors not only supports aesthetic coherence between modes but also marks the games' characters as performances. Musically, the repeated use of several themes, especially during cutscenes, resembles popular cinema's use of music, while the looped background music during gameplay is typical of videogames. Taken together, the audiovisual presentation of the games appears generally designed to "bridge the gaps" between the different modes and to support their perception as a unified whole, while distinctively taking advantage of practices established in film and other earlier media formats.

\section{The comics}

While lacking the games' syncretic (Groensteen 2007, 160) nature, the comics adaptations do not face the games' challenge of needing to unify different presentational modes. Indeed, in stark contrast to the games, the comics' presentation appears to actively undermine such an effect. There are constant switches between different styles, with drawings generally heavily stylized, panels often overflowing with jagged pencil lines, and the coloring sometimes appearing unfinished. The repeated stylistic changes between panels draw attention to the materiality of the comics (Schüwer 
$2008,374)$ as they highlight the existence of the diegetic world as a set of representations. The color palette is monochromatic with color sparsely applied to highlight singular elements. This is most apparent in the use of red against the comics' usual palette of greens, blues, and grays. Another distinctive choice is the use of large soundwords to translate the more hectic diegetic events into a purely visual presentation. The visual presentation more closely resembles that of the games' concept artwork by Yoji Shinkawa than the games themselves. ${ }^{7}$ This combination of stylistic choices amounts to an art brut (Duncan and Smith 2009, 161-162) style suitable for the harsh violent world represented.

That the comics panels are often initially hard to read highlights how the balance between aesthetic and functional needs differs between comics and videogames. Because reception is not internally timed, a comic can afford to include visuals readers might find hard to decipher in a brief moment. Taken together, the presentation stands in noticeable contrast to the comics' narration. This might have been motivated by their status as adaptations, whereby a distinctive presentation can heighten the appeal to potential audiences already familiar with the narrative.

\section{The digital graphic novels}

Simply speaking, the digital graphic novels reinstate the videogames' syncretic nature upon the comics adaptation while retaining a linear narrative. Aside from panels being placed into a strict sequential order, their presentation is altered to achieve a full screen presentation. This is accomplished through the use of film techniques like panning and zooming, whose application also leads to further regulation of individual panels' reading pattern. Furthermore, many panels now feature brief animations as well as gentle "camera" movements, which, combined with a layering of the existing visuals, produces an effect of a less flat diegetic space. An addition unique to the film versions is the presence of credit sequences akin to typical film presentations. In terms of audio, all versions feature a soundtrack that includes new versions of some of the compositions and sound effects taken from the games. Furthermore, the film versions feature all dialogue performed by voice actors, with most of the games' cast reprising their parts. All these choices strengthen the connection between the games and the digital graphic novels by creating a shared aural identity. Noticeably, even in versions with voiced dialogue, the digital graphic novels include speech balloons that remain fixed even when movement is applied to the presentation of the diegetic world. Particularly against the backdrop of the film versions' constant progress, this appears as a marker of the work's comics-specific identity. On the other hand, the version released for the Sony PlayStation Portable highlights the potential to interact with the visuals as the recipient can zoom through image layers while scanning panels. 
In tune with their designation as digital graphic novels, the audiovisual presentation of these adaptations appears to walk the tightrope between presenting how digital media can evolve the experience of reading a comic while simultaneously retaining markers of being a comic. This is the case because the digital graphic novels must identify as comics to justify their existence against other media accessible on the same hardware.

\section{Conclusion}

Looking at Metal Gear Solid, Metal Gear Solid 2: Sons of Liberty, and their adaptations into comics and digital graphic novels reveals distinctive properties of these individual incarnations as well as of the various media formats. For one, they show that both games include a narrative core that remains recognizable throughout its transposition into other media formats. In both games, this core is mostly encompassed inside embedded narrative content and presented through two different modes of presentation. These modes introduce diegetic goals the player's avatar then pursues in another mode through engagement with the game's rule-based systems. In this mode, the player then actively controls the avatar inside emerging narrative events. Together, the structured interplay of these modes forms the general process of playing the games. While this structure is not replicated in the adaptations, it still appears to have had a lasting influence on the process of adaptation. This is because the adaptations prioritize the embedded narrative content that the games present through their film-like cutscenes mode. However, much of the games' lengthy dialogue, their postmodern sensibilities, their references outside of the closer diegetic setting, and their emergent narrative content have been omitted. The adaptations thereby retain many of the games' iconic narrative events but nonetheless feature a distinctively modified narrative. The choice of adapted materials leads to more escapist narratives with a stronger emphasis on physical action.

Both games, despite their general appearance as fixed linear narrations, also offer players various ways to access optional embedded narrative, modify the sequential presentation of narrative events, and even, in a single case, influence the narration's outcome. On these issues, their adaptations, however, show the limitations of their respective media formats. Aside from a single feature in one version of the digital graphic novels, all of these elements had to be removed or fixed into a singular sequence of events. While neither game might be anybody's poster child for nonlinear narration, it is illuminating to see that, despite their rather simple implementation of such features, they create a style of narration unique to videogames and not easily replicated in other forms of media.

In terms of presentation, the games and their adaptations seem to reflect vastly different intentions. The audiovisual presentation of the games appears designed to 'bridge the gaps' between their three modes. By keeping elements such as visual assets and the voiced dialogue consistent throughout all modes, they strive to present themselves as a unified experience. While 
they do not achieve this entirely in terms of their visual presentation, their narration and postmodern sensibilities are able to provide some motivation for the existing inconsistencies. Going into the opposite direction, the comics adaptations' visual presentation actively undermines the perception as a unified experience. Repeatedly switching between different styles, they feature heavily stylized panels overflowing with jagged pencil lines as well as sometimes seemingly unfinished coloring. This presentation also highlights the comics' own materiality, a choice curiously contrasting with the comics' narration completely omitting similar tendencies present in the games. The digital graphic novels, completely reproducing the comics' visuals as well as their text, expand this presentation through the use of cinematic techniques and a new aural presentation. This aural presentation connects these seconddegree adaptations back to the games by referring to it in various ways. The complete reproduction of the comics inside this new multimodal shell of the digital graphic novels shows how the change of media format modifies content even when it is apparently identically reproduced.

In hindsight, the most distinctive and lasting effect of this sequence of adaptations appears to be on two later Metal Gear Solid titles for the Sony PlayStation Portable. Both games featured the participation of the comics' artist Ashley Wood for cutscenes stylistically similar to the digital graphic novels. Apart from this, the success of the comics adaptations appears to have been limited; despite the continued popularity of the videogame series, no further adaptations followed. However, the digital graphic novels appear to have fared even worse. Following the Sony PlayStation Portable version, their feature film versions initially did not even receive a release on Western markets. Their later Western release was then only as part of a collection to celebrate the series' 25 th anniversary, where they found themselves stashed away as a bonus feature on one of the game discs. While it might not be possible to pinpoint a specific reason for the digital graphic novels' lack of success, it is intriguing to consider if and how this relates to their "misfit" nature. All their releases were for technological devices strongly associated with the playback of other forms of media. This might have led to audiences being unsure about the digital graphic novels' nature. Perhaps they might have fared better a few years later when multipurpose devices such as smart phones, tablets, and notebooks brought a distinctive change in how audiences consume media. Predating a general "liberation" of comics from print through digital distribution, the digital graphic novels do not just present a blueprint on how comics can take advantage of this liberation. In their subdued reception, they also present a warning and suggest the potential risks involved when giving up a well-established medial identity.

\section{Notes}

1 The first of these was published in Japan as Metal Gear Solid: Bande Dessinée (2006) and in Europe as Metal Gear Solid: Digital Graphic Novel (2006), while 
the second was only published in Japan as Metal Gear Solid 2: Bande Dessinée (2008), the different titles leading to a markedly different paratextual framing.

2 The distinction between embedded and emergent game narratives goes back to Marc LeBlanc (Salen and Zimmermann 2008, 383). Katie Salen and Eric Zimmerman describe the difference as follows: "Embedded elements are narrative structures directly authored by game designers" while "emergent narrative approaches emphasize the ways that players interact with a game system to produce a narrative experience unique to each player" $(2008,384)$.

3 Holmes calls the three modes active gameplay, cutscenes, and codec sequences. I admit that neither mine nor his term for the player's interactions with the game's rule-based systems is ideal. By itself, gameplay, while often used in popular discourse, has the effect of implying some elements of the game to be not part of the experience of playing. While Holmes's use of active gameplay corrects this effect, it tends to mark the player's engagement with other modes as passive.

4 The degree to which these modes cover many of the narrative developments is even exemplified by Metal Gear Solid itself. After the player has first completed the game, it offers a mode where the narrative is presented through a combination of such embedded elements with brief written summaries.

5 Arguably, despite its title, this means that Metal Gear Solid could also be considered an adaptation of Metal Gear Solid: The Twin Snakes.

6 Here, both games differ from many other contemporary titles in their choice to use neither prerendered backgrounds during gameplay nor prerendered cutscenes. By doing this, the designers opted for a more unified and flexible visual presentation instead of higher quality visuals.

7 A dedication to him is even featured in one of the splash panels of Metal Gear Solid.

\section{Works cited}

Backe, Hans-Joachim. 2008. Strukturen und Funktionen des Erzählens im Computerspiel: Eine typologische Einführung. Würzburg: Königshausen und Neumann.

Bogost, Ian. 2007. Persuasive Games: The Expressive Power of Videogames. Cambridge, MA: MIT Press.

Duncan, Randy, and Matthew J. Smith. 2009. The Power of Comics: History, Form and Culture. New York: Continuum.

Fraction, Matt, and Ashley Wood. 2014. "Metal Gear Solid: Sons of Liberty \#0.” In Metal Gear Solid: Deluxe Edition, 298-327. San Diego: IDW Publishing.

Garner, Alex, and Ashley Wood. 2014. “Metal Gear Solid: Sons of Liberty.” In Metal Gear Solid: Deluxe Edition, 328-593. San Diego: IDW Publishing.

Groensteen, Thierry. 2007. The System of Comics. Jackson: University Press of Mississippi.

Holmes, Dylan. 2012. A Mind Forever Voyaging: A History of Storytelling in Video Games. Self-published.

Hutcheon, Linda. 2006. A Theory of Adaptation. New York: Routledge.

Juul, Jesper. 2005. Half-Real: Video Games between Real Rules and Fictional Worlds. Cambridge, MA: MIT Press. 
Klevjer, Rune. 2002. "In Defense of Cutscenes." In Proceedings of Computer Games and Digital Cultures Conference, edited by Frans Mäyrä, 191-202. Tampere: Tampere University Press.

Metal Gear Solid. 1998. Developed and published by Konami. PlayStation.

Metal Gear Solid: Bande Dessinée. 2006. Developed by Kojima Productions. Published by Konami. PlayStation Portable.

Metal Gear Solid: Digital Graphic Novel. 2006. Developed by Kojima Productions. Published by Konami. PlayStation Portable.

Metal Gear Solid: The Twin Snakes. 2004. Developed by Silicon Knights and Konami. Published by Konami. Nintendo GameCube.

Metal Gear Solid 2: Bande Dessinée. 2008. Developed by Kojima Productions. Published by Konami. DVD.

Metal Gear Solid 2: Sons of Liberty. 2001. Developed and published by Konami. PlayStation 2.

Mukherjee, Souvik. 2015. Video Games and Storytelling: Reading Games and Playing Books. New York: Palgrave Macmillan.

Oprisko, Kris, and Ashley Wood. 2014. "Metal Gear Solid.” In Metal Gear Solid: Deluxe Edition, 4-297. San Diego: IDW Publishing.

Parish, Jeremy. 2018. "Ranking the Metal Gear Series.” Polygon, 23 March. www. polygon.com/features/2018/2/19/17022700/best-metal-gear-games (accessed 31 January 2020).

Parkin, Simon. 2012. “Hideo Kojima: Video Game Drop-Out: Interview Part 1.” The Guardian, 24 March. www.theguardian.com/technology/gamesblog/2012/may/ 23/hideo-kojima-interviewpart-1 (accessed 31 January 2020).

Parkin, Simon. 2017. An Illustrated History of 151 Video Games. London: Lorenz Books.

Ryan, Marie-Laure, and Jan-Noël Thon. 2014. "Storyworlds across Media: Introduction." In Storyworlds across Media: Toward a Media-Conscious Narratology, edited by Marie-Laure Ryan and Jan-Noël Thon, 1-21. Lincoln: University of Nebraska Press.

Salen, Katie, and Eric Zimmerman. 2008. Rules of Play: Game Design Fundamentals. Cambridge, MA: MIT Press.

Schüwer, Martin. 2008. Wie Comics erzählen: Grundriss einer intermedialen Erzähltheorie der grafischen Literatur. Trier: Wissenschaftlicher Verlag Trier. 